\title{
Electromagnetic Power Harvester Using Wide-Angle and Polarization-Insensitive Metasurfaces
}

\author{
Xuanming Zhang, Haixia Liu * and Long $\mathrm{Li}^{*}$ (1) \\ Key Laboratory of High Speed Circuit Design and EMC of Ministry of Education, School of Electronic \\ Engineering, Xidian University, Xi'an 710071, China; zhd9988@163.com \\ * Correspondence: hxliu@xidian.edu.cn (H.L.); lilong@mail.xidian.edu.cn (L.L.); Tel.: +86-029-8820-4458 (H.L.); \\ +86-029-8820-1157 (L.L.)
}

Received: 16 February 2018; Accepted: 22 March 2018; Published: 26 March 2018

\begin{abstract}
A new wide-angle and polarization-insensitive metasurface (MS) instead of traditional antenna is built as the primary ambient energy harvester in this paper. The MS is a two-dimensional energy harvesting array that is composed of subwavelength electrical small ring resonator that is working at $2.5 \mathrm{GHz}$ (LTE/WiFi). In the case of different polarization and incidence angles, we demonstrate the metasurface can achieve high harvesting efficiency of $90 \%$. The fabricated prototype of $9 \times 9$ MS energy harvesting array is measured, and the experimental results validate that the proposed MS has a good performance more than $80 \%$ of energy harvesting efficiency for arbitrary polarization and wide-angle incident waves. The good agreement of the simulation with the experiment results verifies the practicability and effectiveness of the proposed MS structure, which will provide a new source of supply in wireless sensor networks (WSN).
\end{abstract}

Keywords: metasurface (MS); energy harvesting; wide-angle; polarization-insensitive

\section{Introduction}

With the rise of the internet of things (IoT) technology, the micro wireless sensor networks (WSN) are being extensive development. In recent years, with the development of the ultra-low power consumption chip technology, the power consumption of wireless sensor node has already entered a microwatt magnitude range. At the same time, an increasing number of wireless network and mobile communication base stations and other radio transmitting equipment that is full of rich electromagnetic energy in the surrounding environment. It is also a research hotspot to reduce the demand of the battery by absorbing the ambient energy to power a sensor node automatically and adaptively in the electromagnetic environment.

Current researches show that there are about 12 million wireless communication base stations in the global in 2012, about 68 billion equipments using a radio frequency identification card (RFID) in 2013, and the world has about 40 billion WiFi consumer electronic equipments in 2014 [1]. Figure 1 shows that the ambient power spectrum distribution of the electromagnetic field measured in the campus environment of Xidian University, China. We can find that the ambient energy peaks appear in the existing wireless communication frequency bands. These micro electromagnetic energies that distribute in the environment are expected to be used again after harvesting efficiently.

Metamaterials are the artificial composite structures or composite materials with superior physical properties that some natural materials do not have. Metasurfaces (MS) are two-dimensional metamaterial structures, which have been attracting more attention, due to a wide range of potential applications for wireless power transfer and harvesting [2]. A perfect metamaterial absorber has been firstly proposed in 2008 by Landy et al. [3]. Different from the electromagnetic metamaterial absorber [4-6], the small split-ring resonators (SRR) were first used to collect energy in 
2012 [7]. After that, some other SRRs [8-10], electric-inductive-capacitive (ELC) resonators [11], and ground-backed complementary split-ring (G-CSRR) resonator have also been proposed for electromagnetic energy harvesting [12,13]. The recently reported wideband ground-backed complementary split-ring (WG-CSRR) resonator structure can improve the bandwidth [14]. Each unit in the WG-CSRR structure requires four ports in order to collect energy at the same time.

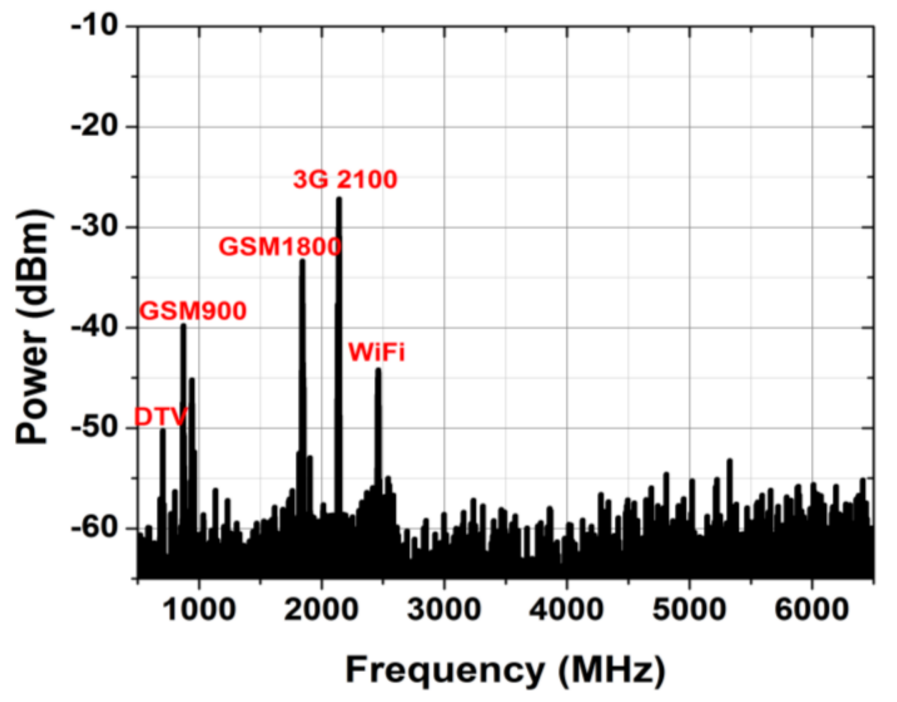

Figure 1. Power spectrum distribution measured in the campus of Xidian University, China.

This paper uses a new and simple electromagnetic MS as the ambient energy harvester in the wireless communication systems. The proposed MS is a two-dimensional energy harvesting array that is composed of subwavelength electrical small square-ring resonator unit. Because the incident angles and polarizations of environmental electromagnetic waves are random and unknown, the conventional energy harvesting antenna or polarization-related metamaterial harvester cannot maintain the high collection efficiency for arbitrary direction and polarization. No matter whether the incident wave is transverse electric (TE) or transverse magnetic (TM) polarization mode, the proposed MS unit or array can keep a high RF power harvesting efficiency. Due to the frequency distribution of electromagnetic energy in the environment has the narrow-band characteristics, this work uses only one harvesting port designed at the appropriate location for spatial filtering, which can simplify the back-end matching and rectifier circuit complexity, and realize the high efficiency of energy harvesting. The proposed MS is sub-wavelength, wide-angle, and polarization-insensitive, which is suitable for ambient power harvesting.

\section{Design of the MS Energy Harvester}

In the energy harvesting system, the rectenna is the most critical part [15-17]. It can be divided into energy harvester (e.g., antenna) and rectifier circuit. As the primary energy collector, it receives electromagnetic RF energy, which is converted to DC power through a rectifier circuit. The energy harvesting using MS should meet the following two conditions: (a) The electromagnetic wave energy can be captured by the MS structure as much as possible; (b) The captured energy into the MS structure can effectively be transferred into the rectifier circuits (matching load).

This paper builds a two-dimensional energy harvesting array that is composed of sub-wavelength square-ring MS, and each small unit can be equivalent to LC resonance circuit. The incident wave is stimulated to produce surface current on the surface of the MS structure. Similar to the antenna-based coaxial feed, we take a via hole in the unit with an appropriate place and then lead the surface current to the port that is connected to the resistive load [11]. The MS energy collector can capture electromagnetic wave energy and transfer to the load, rather than dissipate in the structure. The designed MS structure 
is shown in the Figure 2. The center working frequency is set to $2.5 \mathrm{GHz}$ (LTE/WiFi). The upper layer is the square-ring array, and the middle is the low-loss dielectric layer and the bottom layer is the metallic ground. The energy harvesting ports are set at the corner of each ring.

Using ANSYS HFSS to establish the numerical simulation model, the boundary condition of the unit cell is set as periodic boundary conditions (PBC), which is combined with the Floquet ports to calculate the infinite array model. The port excitation of the incident wave power is set to $1 \mathrm{~W}$. The dielectric substrate is F4B that has relative permittivity of 2.65 and a dielectric loss tangent of 0.001. The dimensions of the square-ring element are as follows: $p=15.7 \mathrm{~mm}, l=15 \mathrm{~mm}, w=0.8 \mathrm{~mm}$, and $t=3 \mathrm{~mm}$. Figure 3 shows that the calculated efficiency of the designed MS array for various load resistance at the operating frequency of $2.5 \mathrm{GHz}$. It can be seen that the highest efficiency of the operating frequency can be simultaneously obtained when the load resistance is $500 \Omega$. It should be noted that this matching load value is a local optimal solution. Other parameters, such as MS structure size, dielectric constant, and thickness of substrate, etc., will also affect the matching load value and the operating frequency. By changing these parameters, we can flexibly design the matching load value of rectifier circuit. This port is located in the diagonal direction of the square-ring element. Note that we can flexibly change the working frequency band, matching the load value by changing the value of each parameter of the MS structure.
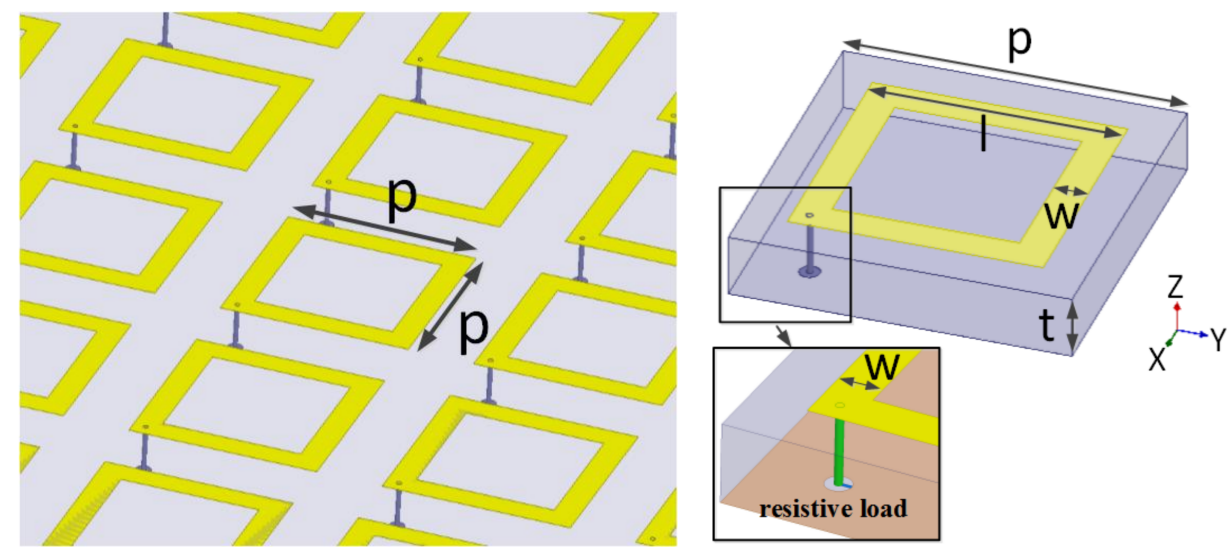

Figure 2. The square-ring resonator metasurface (MS) structure for ambient power harvesting.

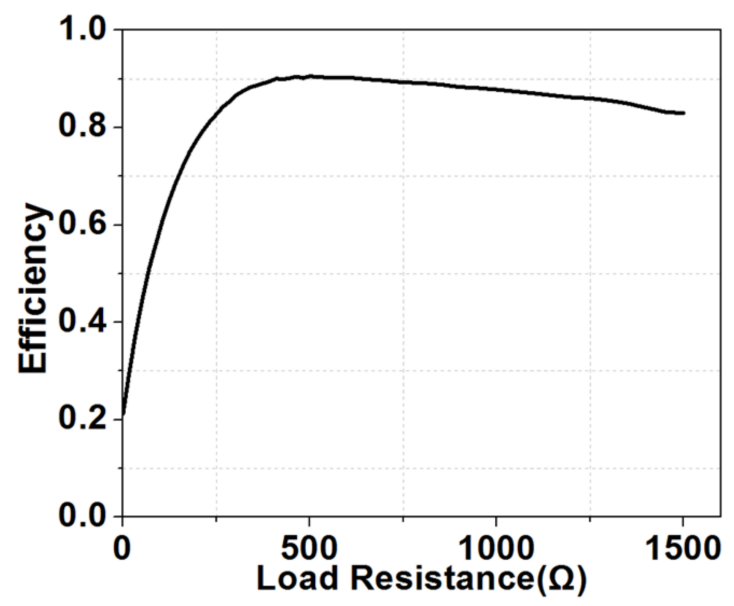

Figure 3. Calculated efficiency of the designed MS array for various values of load resistance at the operating frequency of $2.5 \mathrm{GHz}$. 
When compared to the periodic dimension of the previous works, such as the SRR element, is about $1 / 4$ operating wavelength at $5.8 \mathrm{GHz}$ [7], and the G-CSRR element is about $1 / 3$ operating wavelength at $5.55 \mathrm{GHz}$ [13], and the WG-CSRR element is about $1 / 5$ operating wavelength at $5.6 \mathrm{GHz}$ [14], the proposed design in this paper is about $1 / 8$ operating wavelength at $2.5 \mathrm{GHz}$, which is more miniaturization. Note that the proposed closed square-ring resonant ring rather than the split-ring form from existing literature is adopted in this paper. In spite of the angles and polarizations of the incident wave, two symmetrical annular currents in the same direction excited by an incident wave on the surface of the closed-ring can channel to the collection port. The open-ended surface currents distribution that was generated by various incident waves from the split-ring resonant lead to single-port collection with angle and polarization sensitive performance on a split-ring structure. So, the proposed square-ring MS structure can harvesting wide-angle, polarization-insensitive incident waves by optimizing the position of the harvesting point, and maintain high efficiency when compared to the structure described in the reference [6,10-12] only collects single-polarized incident waves. By changing the value of each parameter of the MS structure $\left(L, W, P, \varepsilon_{r}, t\right)$, we can flexibly change the working frequency band, matching the load $(R)$ value, to achieve the performance of effective energy harvesting. The square-ring MS structure is proposed to replace the traditional antenna as the front of the rectifier in the energy-harvesting system. It is worth pointing out that the proposed MS structure can effectively captures the ambient radiofrequency energy and then concentrated on the load resistance, which can be replaced by the rectifier circuit in the later work.

The power harvesting efficiency of the MS collector can be computed by

$$
\eta=\frac{P_{L}}{P_{r}} \times 100 \%
$$

where $P_{L}$ is the power collected by the load, and the $P_{r}$ is the received total power incident on the cross-sectional area (A) of the whole MS structure, which can be computed using the surface integral of a Poynting vector on A.

The simulation results of the reflection coefficient $S_{11}$ and power harvesting efficiency are shown in Figure 4 . It can be seen that the impedance matching is good at the working frequency of $2.5 \mathrm{GHz}$. That is to say, the MS structure has the ability of capturing the electromagnetic wave energy. By calculating the receiving power in the lumped port, we can see that the MS harvester can collect the energy more than $90 \%$ from the incident power at $2.5 \mathrm{GHz}$. The captured electromagnetic energy by the square-ring MS can be transferred into the matching load through vias effectively. Figure 5 is the surface current distribution on the square ring and the back ground of the MS structure at $2.5 \mathrm{GHz}$. It can be seen that the surface current flows along the small ring, and the energy is eventually harvested to the port of the resistive load.

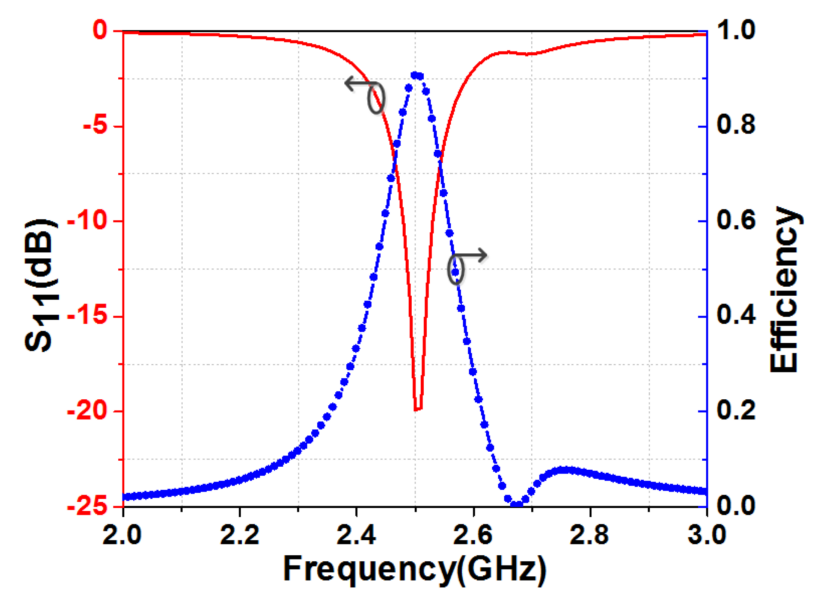

Figure 4. Reflection coefficient $S_{11}$ and power harvesting efficiency using square-ring MS structure. 
The incident angle and polarization stability of MS structure should be considered from the two cases of transverse electric (TE) and transverse magnetic (TM) oblique incidences. The TE-polarized oblique incidence refers to the change of electromagnetic wave incident angles to ensure the electric field vector is always parallel with the surface of MS structure, while the TM-polarized oblique incidence refers to the change of the electromagnetic wave incident angles to ensure that the magnetic field vector is always parallel with the surface of MS structure. Figure 6a shows the power harvesting efficiency characteristics at oblique incidence with TE polarization in the resonant bands. When the harvester tilted with angle $\theta=0^{\circ}$ and $15^{\circ}$, the maximum harvesting efficiency achieved was $90.5 \%$ at $2.5 \mathrm{GHz}$ and $90.4 \%$ at $2.52 \mathrm{GHz}$. For angle $\theta=30^{\circ}$ and $45^{\circ}$, collection efficiency of $89.8 \%$ at $2.56 \mathrm{GHz}$ and $88.4 \%$ at $2.62 \mathrm{GHz}$. The last tilted angle is $\theta=60^{\circ}$ and a collection efficiency of $85.2 \%$ is achieved at $2.67 \mathrm{GHz}$. For TM polarization, as shown in Figure $6 \mathrm{~b}$, the energy collection efficiency is $90 \%$ (when $\theta=0^{\circ}$ at $2.5 \mathrm{GHz}$ ), and the maximum efficiency is $91.4 \%$ (when $\theta=45^{\circ}$ at $2.62 \mathrm{GHz}$ ). When the oblique incidence $\theta$ are $15^{\circ}, 30^{\circ}$, and $60^{\circ}$, the harvesting efficiency are $91.1 \%$ at $2.52 \mathrm{GHz}$, $91 \%$ at $2.56 \mathrm{GHz}$, and $91.2 \%$ at $2.67 \mathrm{GHz}$. The rate of change of efficiency is very small. As the angle of incidence waves increases, the working frequency of the maximum efficiency obtained by the MS will slight shift. The MS have a certain bandwidth. No matter TE or TM oblique incidence from $0^{\circ}$ to $60^{\circ}$, the energy harvesting efficiency using the square-ring MS collector is always stable and the collection efficiency can reach $90 \%$. Though it has a certain frequency-shift in the incidence of the large angles, it can be adjusted through backend self-adaptive tuning circuits.

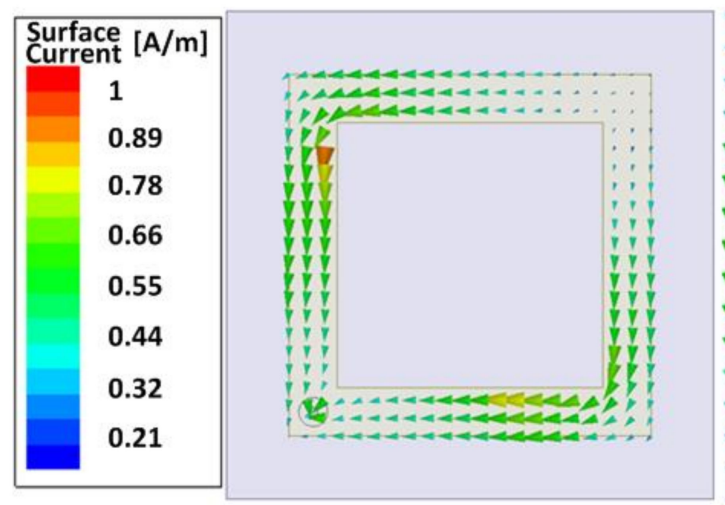

(a)

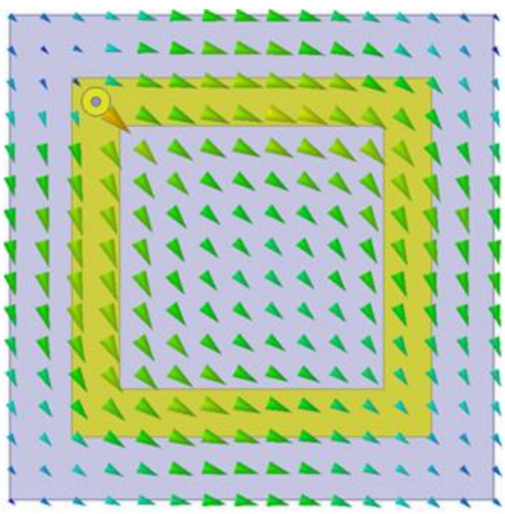

(b)

Figure 5. Surface current distribution on the (a) square ring and (b) back ground of the MS structure at the resonant frequency $(2.5 \mathrm{GHz})$.

The surface current analysis can be used to gain insight into the polarization and the angle stability of the MS. The resonant behavior can be described by using the equivalent inductance $(\mathrm{L})$ and the equivalent capacitance (C) of the MS structure. The small square-ring of each unit and gap between units can be equivalent to LC resonance circuit. The surface currents induced on the front metallic layer operating at work frequency of $2.5 \mathrm{GHz}$ are oscillated along the square ring of each element and transferred to the load through vias. The surface currents in TE and TM polarization of the incident wave at the resonant frequency $(2.5 \mathrm{GHz})$ and non-resonant frequency $(2.2 \mathrm{GHz})$ for the incidence angle $0^{\circ}$ are shown in Figure 7, respectively. It can be seen that the maximum intensity of the surface induced current to the load is generated on the metallic ring at operating frequency of $2.5 \mathrm{GHz}$, and the maximum energy harvesting efficiency can be obtained. When offsetting the operating frequency, the intensity of the surface induced current on the metallic ring is reduced, which causes the load absorption decreased. Figure 8 shows the surface current distribution at the working frequency for different incidence angles in TE polarization. Maximum energy harvesting efficiency with $\theta=30^{\circ}$ and $60^{\circ}$ of incident wave at $2.56 \mathrm{GHz}$ and $2.67 \mathrm{GHz}$, respectively. Because the square-ring MS is centrosymmetric, no matter whether the incident wave is TE or 
TM polarization with large angle range, it can excite surface currents along the metal ring and translate into load by vias for efficient energy harvesting.

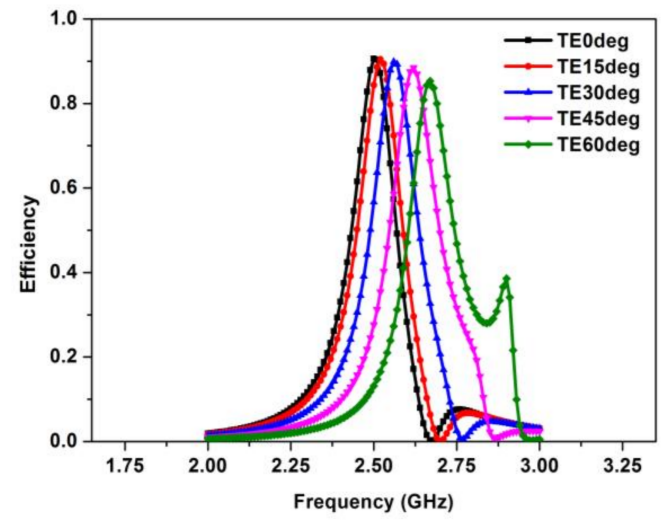

(a)

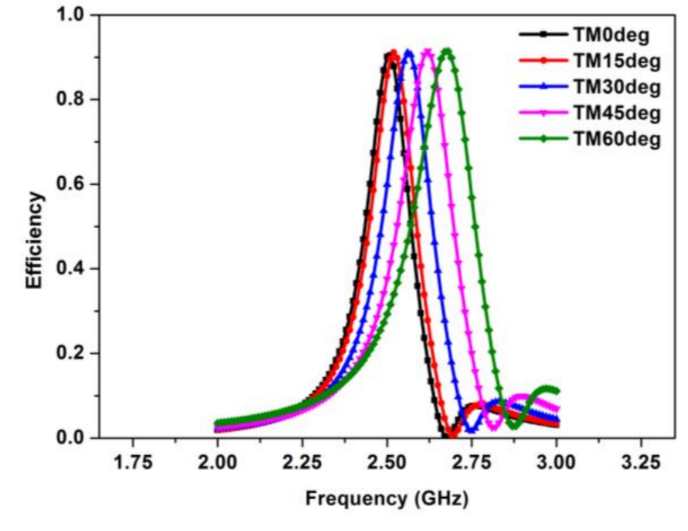

(b)

Figure 6. Power harvesting efficiency of (a) transverse electric (TE)-polarized oblique incidence and (b) transverse magnetic (TM)-polarized oblique incidence.
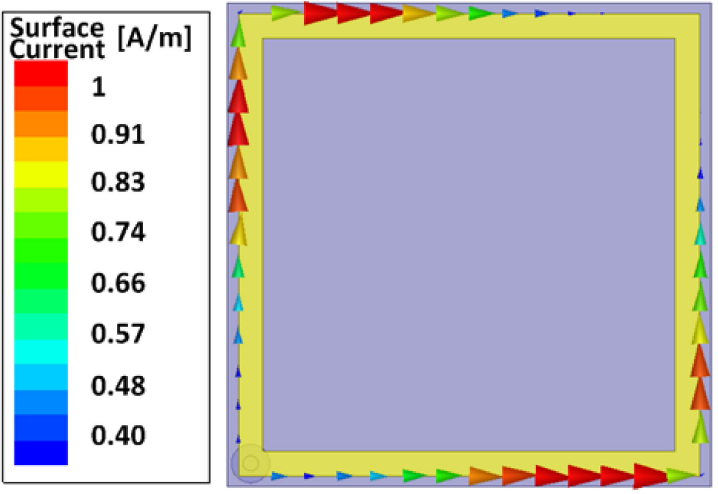

(a)
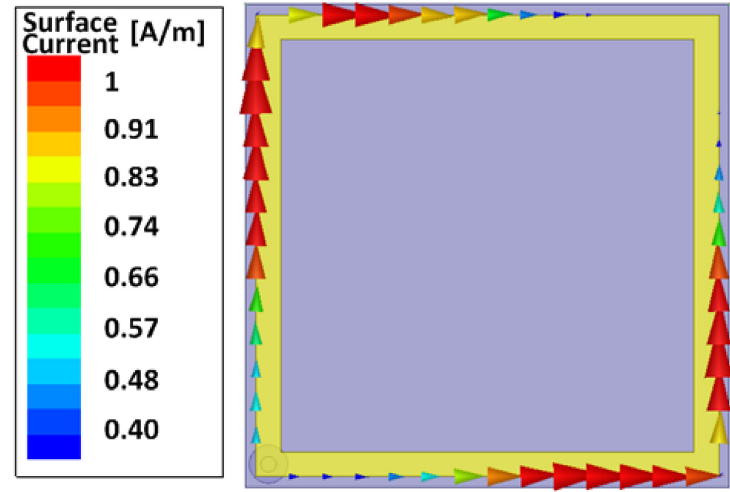

(c)

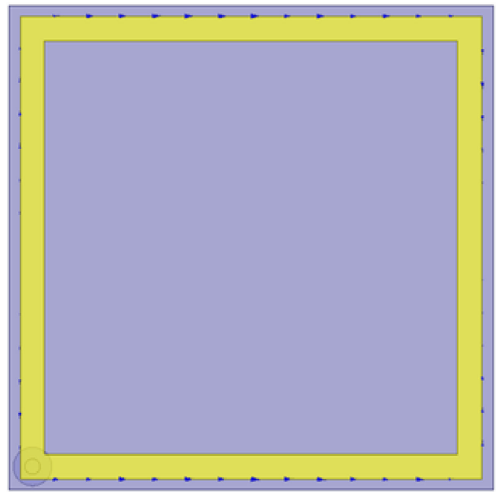

(b)

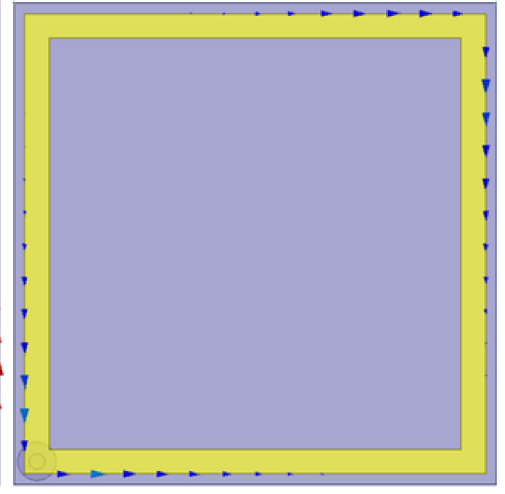

(d)

Figure 7. Simulated surface current distribution on the square-ring at (a) the resonant frequency of $2.5 \mathrm{GHz}$ with TE polarization; (b) the non-resonant frequency of $2.2 \mathrm{GHz}$ with TE polarization; (c) $2.5 \mathrm{GHz}$ with TM polarization; and (d) $2.2 \mathrm{GHz}$ with TM polarization. 


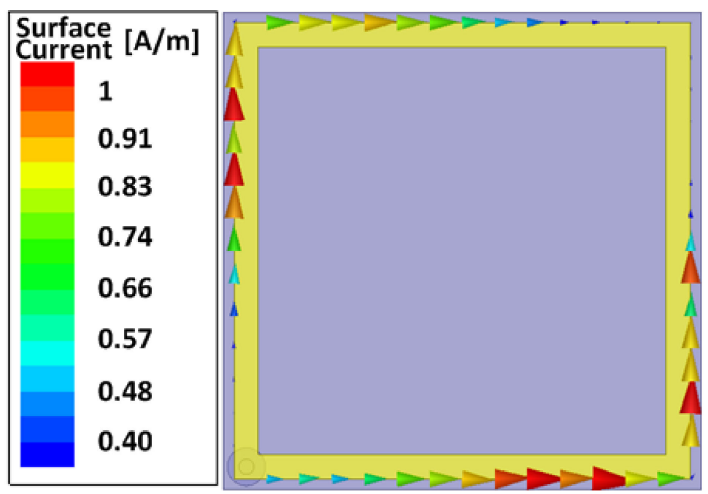

(a)

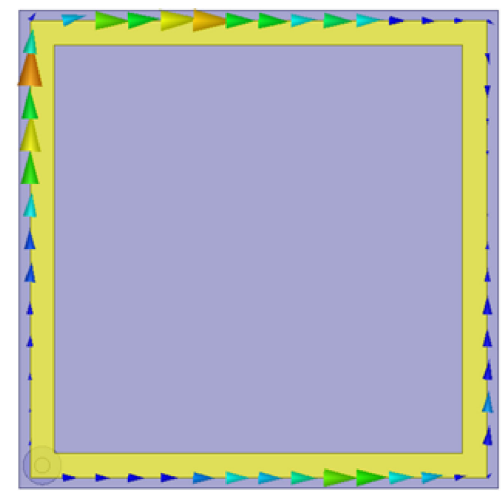

(b)

Figure 8. Simulated surface current distribution on the square-ring with different incidence angles (a) $\theta=30^{\circ} ;$ (b) $\theta=60^{\circ}$.

\section{Experimental Verification}

In this section, a $9 \times 9$ MS energy harvesting array has been fabricated to verify the performance, as shown in Figure 9. The experimental scheme is illustrated in Figure 10. The MS energy harvester placed in the far field of the horn antenna that excited by a signal generator at the power level of $14 \mathrm{dBm}$, to ensure that the electromagnetic wave incident to the MS structure is a plane wave excitation [11]. The power of the load resistance on the MS structure is tested by using a spectrum analyzer. Because each cell of the $9 \times 9$ MS array has a harvesting port to collect energy at the same time, the power measurement for all of the ports is almost impossible. The central unit can represent other units in a large array without edge effects. Therefore, a good estimation of the power harvesting can be measured. Based on the analysis and discussion above, we only test energy harvesting efficiency of the center element, and the overall efficiency of array can be calculated by multiplying the efficiency of the center element by the number of array elements [13].

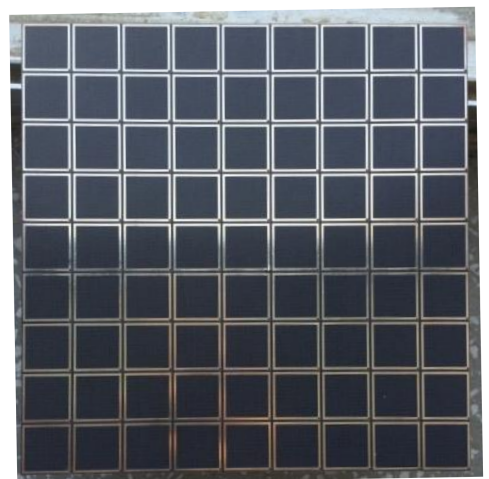

(a)

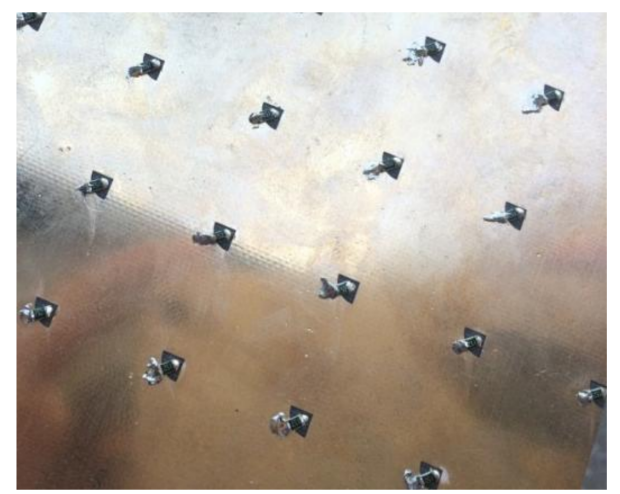

(b)

Figure 9. Photograph of the fabricated MS structure array on (a) front view (b) back view.

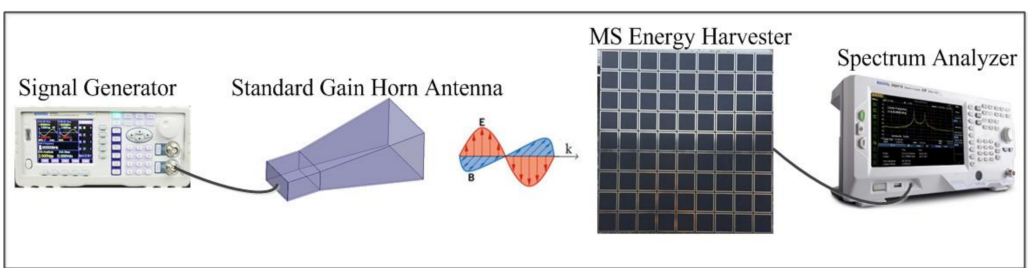

Figure 10. Experimental setup for measuring the power harvesting efficiency of MS structure. 
The energy collection efficiency is given by:

$$
\eta=\frac{P_{R F}}{P_{M S}} \times 100 \%
$$

where $P_{R F}$ is the total time-average power harvested by the port of central element (measured by the spectrum analyzer). $P_{M S}$ is the total time-average power incidence on the center cell of the MS by the standard gain horn antenna.

Figure 11 shows the measured energy collection efficiency of the fabricated $9 \times 9$ MS energy harvesting array in different angles of TE and TM oblique incidence from the same distance. It can be seen that the collected energy efficiency is more than $80 \%$ in the TE normal incidence at $2.58 \mathrm{GHz}$. When the incident angle is changed to $\theta=60^{\circ}$, the energy collection efficiency in this case is still $81 \%$, but the operational frequency will shift to $2.7 \mathrm{GHz}$. In the TM polarization, we can see that the maximum collection efficiency is $85.1 \%$ and the resonant frequency is $2.57 \mathrm{GHz}$ when the incident angle $\theta$ is $0^{\circ}$. When the incident angle changes to $\theta=60^{\circ}$, the resonant frequency of maximum efficiency shifts to $2.69 \mathrm{GHz}$, and the collection efficiency is $82.6 \%$. It is verified that the proposed MS structure has a good performance on the incident electromagnetic wave with arbitrary polarization. The energy harvesting efficiency and the operational frequency that was obtained from the measurement and the numerical analysis are different by reason of typical fabrication tolerances and measurement error. Moreover, the fabricated array is a $9 \times 9$ array when compared to infinite periodic array in the simulation. So, the measurement efficiency is lower than the simulation and the operating frequency have a slight offset, but the energy collection efficiency remains above $80 \%$. The frequency shift can be adjusted using self-adaptive tuning circuits. The good agreement of the simulation with the experiment results verifies the practicability and the effectiveness of the proposed MS structure.

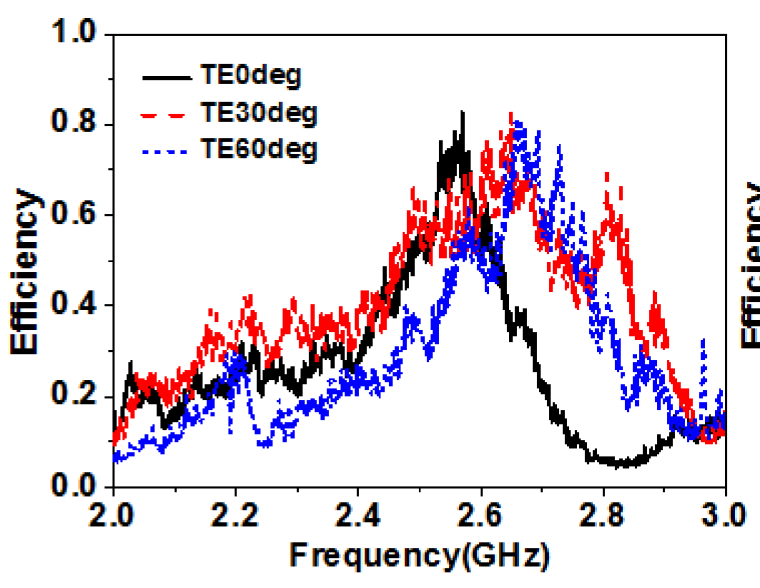

(a)

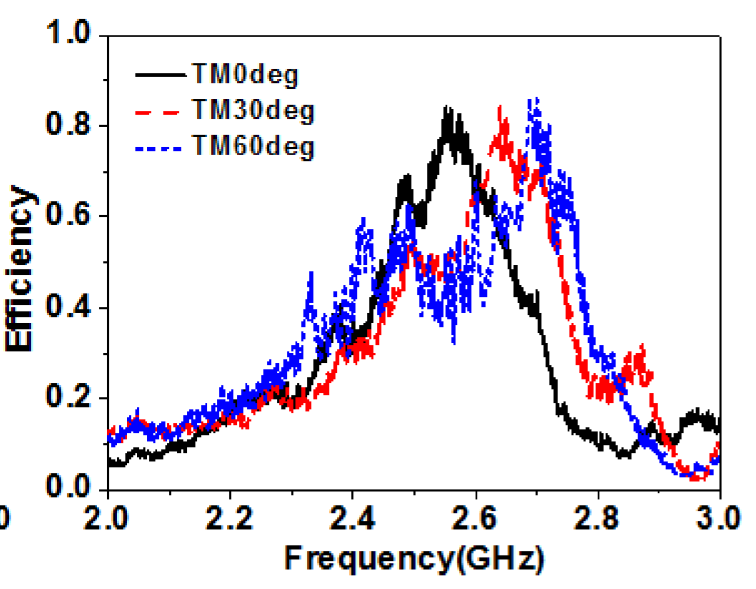

(b)

Figure 11. Measured energy harvesting efficiency of the fabricated $9 \times 9$ MS energy harvesting array for different polarization and incident angles, (a) TE-polarized oblique incidence and (b) TM-polarized oblique incidence.

\section{Conclusions}

In this paper, a new polarization-insensitive MS was designed, fabricated, and experimentally demonstrated to absorb the electromagnetic power in wide incidence angle. The designed MS is an electrical small square-ring resonator whose center operating frequency is $2.5 \mathrm{GHz}$ (LTE/WiFi). No matter whether the incident wave is TE or TM polarization, the proposed MS can keep high RF power harvesting efficiency. The good performance and accuracy of the proposed MS design are verified by using simple and effective simulations and measurements. The proposed square-ring MS 
harvester has many advantages, such as miniaturization, polarization insensitivity, wide angle stability, high efficiency, and easy to conformal, which are suitable for ambient power harvesting.

Acknowledgments: This work is supported by National Key R\&D Program of China, and is supported by National Natural Science Foundation of China under Contract No. 51477126, and supported by Technology Explorer and Innovation Research Project.

Author Contributions: All authors contributed substantially to the reported work. Long Li conceived the idea of metasurface for ambient energy harvesting. Xuanming Zhang designed the subwavelength electrical small ring resonator and performed the simulations and experiments; Haixia Liu provided the main instructions of experiments; Long Li provided the main instructions of this study and revised the paper.

Conflicts of Interest: The authors declare no conflict of interest.

\section{References}

1. Hemour, S.; Wu, K. Radio-frequency rectifier for electromagnetic energy harvesting: Development path and future outlook. Proc. IEEE 2014, 102, 1667-1691. [CrossRef]

2. Li, L.; Liu, H.; Zhang, H.; Xue, W. Efficient wireless power transfer system integrating with metasurface for biological applications. IEEE Trans. Ind. Electron. 2018, 65, 3230-3239. [CrossRef]

3. Landy, N.I.; Sajuyigbe, S.; Mock, J.J. A perfect metamaterial absorber. Phys. Rev. Lett. 2008, 100, 207402. [CrossRef] [PubMed]

4. Ding, F.; Cui, Y.; Ge, X.; Jin, Y.; He, S. Ultra-broadband microwave metamaterial absorber. Appl. Phys. Lett. 2012, 100, 103506. [CrossRef]

5. Luukkonen, O.; Costa, F.; Monorchio, A.; Tretyakov, S.A. A Thin Electromagnetic Absorber for Wide Incidence Angles and Both Polarizations. IEEE Trans. Antennas Propag. 2009, 57, 3119-3125. [CrossRef]

6. Li, L.; Yang, Y.; Liang, C. A wide-angle polarization-insensitive ultra-thin metamaterial absorber with three resonant modes. J. Appl. Phys. 2011, 110, 063702. [CrossRef]

7. Ramahi, O.M.; Almoneef, T.S.; AlShareef, M.; Boybay, M.S. Metamaterial particles for electromagnetic energy harvesting. Appl. Phys. Lett. 2012, 101, 173903. [CrossRef]

8. Hawkes, A.M.; Katko, A.R.; Cummer, S.A. A microwave metamaterial with integrated power harvesting functionality. Appl. Phys. Lett. 2013, 103, 163901. [CrossRef]

9. Alshareef, M.R.; Ramahi, O.M. Electrically small resonators for energy harvesting in the infrared regime. J. Appl. Phys. 2013, 114, 223101. [CrossRef]

10. Shang, S.; Yang, S.; Liu, J. Metamaterial electromagnetic energy harvester with high selective harvesting for left- and right-handed circularly polarized waves. J. Appl. Phys. 2016, 120, 045106. [CrossRef]

11. Almoneef, T.; Ramahi, O.M. Metamaterial electromagnetic energy harvester with near unity efficiency. Appl. Phys. Lett. 2015, 106, 153902. [CrossRef]

12. Alavikia, B.; Almoneef, T.S.; Ramahi, O.M. Electromagnetic energy harvesting using complementary split-ring resonators. Appl. Phys. Lett. 2014, 104, 163903. [CrossRef]

13. Alavikia, B.; Almoneef, T.S.; Ramahi, O.M. Complementary split ring resonator arrays for electromagnetic energy harvesting. Appl. Phys. Lett. 2015, 107, 033902. [CrossRef]

14. Alavikia, B.; Almoneef, T.S.; Ramahi, O.M. Wideband resonator arrays for electromagnetic energy harvesting and wireless power transfer. Appl. Phys. Lett. 2015, 107, 243902. [CrossRef]

15. Chen, Z;; Guo, B.; Yang, Y.; Cheng, C. Metamaterials-based enhanced energy harvesting: A review. Phys. B Condens. Matter 2014, 438, 1-8. [CrossRef]

16. Olgun, U.; Chen, C.C.; Volakis, J.L. Design of an efficient ambient WiFi energy harvesting system. IET Microw. Antennas Propag. 2012, 6, 1200-1206. [CrossRef]

17. Chin, C.H.K.; Xue, Q.; Chan, C.H. Design of a 5.8-GHz rectenna incorporating a new patch antenna. IEEE Antennas Wirel. Propag. Lett. 2005, 4, 175-178. [CrossRef] 